\title{
Short-Term Photovoltaic Output Forecasting with Weakly Related Meteorological Data
}

\author{
C.H. Lin, Y. Xiao, J.G. Chen, X.K. Wen \\ Guizhou Electric Power Test \& Research Institute \\ China
}

\author{
C. Du \\ North China Electric Power University \\ China
}

\begin{abstract}
Photovoltaic (PV) output is influenced by many meteorological factors. The significant degree of meteorological data influences the accuracy of forecasting result. This paper proposed a short-term PV output forecasting method while the weather data and PV output data were weak correlated. By analyzing meteorological historical data and PV output historical data, the main factors effecting PV generation were found out by Pearson correlation coefficient. Based on relevant factors, fuzzy clustering analysis method was used to select similar days, and then support vector regression (SVR) forecasting model was built. SVR model has excellent learning ability for small sample. To determine model parameters, a two-step method was proposed. First, using the global search method to determine the value of parameter $\varepsilon$ and the appropriate range of kernel parameter $p$ and regularization parameter $\mathrm{C}$, then using self-adaptive differential evolution algorithm to find the optimal $p$ and $C$, in order to improve the forecast accuracy when parameter $\varepsilon$ was selected in large scale. Examples show that the method proposed in this paper has good forecasting ability when the weather data and PV output data are weak correlated.
\end{abstract}

Keywords-distributed photovoltaic; PV output forecasting; support vector machine; parameter selected

\section{INTRODUCTION}

Clean energy represented by solar energy attracts more and more widespread attention. After large-scale PV plants connect to the grid, multiple effects on safe, economic and reliable operation will be emerged [1].So it is necessary to forecast the short-term PV output[2], in order to control it actively.Output of roof-mounted PV power systems is always forecasted by statistical method.Statistical method relies on historical record to build forecasting models, predicting output directly.

Reference[3] proposes a method to forecast roof-mounted PV system output by artificial neural network (ANN).Reference[4]describes an algorithm based on weather patterns. Firstly, divide historical samples into subclasses by empirical mode decomposition, and then use support vector machine (SVM) to forecast PV output in the different subclasses. Reference [5] forecasts short-term small-scale PV plant output by the use of season time series model and SVM method.

The literatures show that statistical for ecastingmore depends on meteorological data where the PV plant is located. For traditional statistical forecasting, only when the number of samples is sufficiently large, the algorithm performance can be the oreticallyguaranteed. But the meteorologicalmeasurement instrumentsare still not perfect in China, so they cannot provideprecise enough information to do forecasting. Even if the PV plantsuse the same meteorological information measured by one device nearby can also cause problems such as the correlation to the output data is weak, the noise in the sample is large, so the number of effective samples is reduced. The applicability of traditional statistical method is lessening.

To overcome the disadvantage that less correlation between meteorological dataanddistributedPV outputdata, we use support vector regression (SVR) model to for ecastitsoutput. First, fuzzy clustering analysis (FCA) was used to extract similar days. Subsequently, support vector regression model which has good predictionability for smallsample was established. Then a two-step method which combines globalgrid search (GS) algorithm and self-adaptive differential evolution (SADE) algorithm was proposed to select optimal parameters, forming a short-term PV output forecasting method called FCA-GS/SADE-SVR method. Finally, apractical example was tested toverify the effectiveness of the proposed method.

\section{STRONG CORRELATION SAMPLE CONSTRUCTION}

\section{A. Characteristics of Short-Term PV Output}

PV output is affected by many factors [6-7] like the intensity of solar radiation, solar incidence angle, angle of the solar cell module installed, temperature, wind speed, cloud amount, dust amount, shadow, etc. For a given PV system, relevance of PV output and external factors are inherent in the historical data [8]. Therefore, through the study of historical data, the ability to predict the future information can be obtained.

\section{B. Data Pre-Processing}

The roof-mounted PV power plant in this article locates at longitude $106^{\circ} 07^{\prime} \sim 107^{\circ} 17^{\prime}$, between latitude $26^{\circ} 11^{\prime} \sim 27^{\circ} 22^{\prime}$. As used herein, the PV output data are taken from the roofmounted PV power plant actual record from February 1, 2013 to March 31, 2013, while the sample time is $10 \mathrm{~min}$. Wind speed and temperature data are from the local numerical weather forecasting, time resolution is 1h. Because solar radiation intensity measuring devices are not yet universal, so we use HOMER software to simulate radiation intensity in hours. Thus, the test data have a gap between real situation, and correlation between output data is weak.

Normalized all data to eliminate the impact of different dimensions. The formula is as follows: 


$$
x=\frac{x^{\prime}-x_{\min }^{\prime}}{x_{\max }^{\prime}-x^{\prime}}
$$

Where: $x^{\prime}$ are the realvalue of PVoutput, light intensity, temperature, and wind speed; $\mathrm{x}_{\max }^{\prime}$ arethe maximumPVoutput, light, temperatureand wind speed in all samples; $\mathrm{x}_{\min }^{\prime}$ are the minimumoutput,light, temperatureand wind speed; $x$ are the normalizedvalue.

We use the most widely usedPearsoncorrelationcoefficient methodtoanalyze the relationship betweenthe PVoutput and light intensity, temperature, wind speed.

SettwovariablesX and $\mathrm{Y}$, and each group of samples is expressed as $\left(X_{i}, Y_{i}\right) \quad(i=1,2, \cdots, n)$, the Pearson linearcorrelation coefficient formula is:

$$
\hat{\rho}=\frac{\sum_{i=1}^{n}\left(X_{i}-\bar{X}\right)\left(Y_{i}-\bar{Y}\right)}{\sqrt{\sum_{i=1}^{n}\left(X_{i}-\bar{X}\right)^{2}} \sqrt{\sum_{i=1}^{n}\left(Y_{i}-\bar{Y}\right)^{2}}}
$$

Where, $\bar{X}=\frac{1}{n} \sum_{i=1}^{n} X_{i}, \quad \bar{Y}=\frac{1}{n} \sum_{i=1}^{n} Y_{i}$.

According to the historical datawithin 2 months,the correlation coefficients of eachmeteorological factorsassociated withPVoutput are calculated, shown in Table1.

TABLE I. CORRELATION COEFFICIENTS BETWEEN PV OUTPUT DATA AND METEOROLOGICAL DATA.

\begin{tabular}{|c|c|c|c|}
\hline $\begin{array}{c}\text { Meteorological } \\
\text { factor }\end{array}$ & $\begin{array}{c}\text { Solar } \\
\text { irradiance }\end{array}$ & Temperature & $\begin{array}{c}\text { Wind } \\
\text { speed }\end{array}$ \\
\hline $\begin{array}{c}\text { Correlation } \\
\text { coefficient }\end{array}$ & 0.55 & 0.64 & 0.13 \\
\hline
\end{tabular}

The two variables will have higher degree of linear relationship if their correlation coefficient closer to 1.When $|\hat{\rho}| \geq 0.8$ verification of weak correlation among samples.

Choose relatively strong correlated factors: light intensityand temperature, as the mainfactors affecting thephotovoltaic power generation, building strong correlatedsmall-sample.

\section{Similar Days Selected By Fuzzy Clustering Algorithm}

By appropriate screening and classification, it can improve the similarity between samples and improve accuracy of forecasting. Fuzzyclustering algorithm is used to selectsimilar days in this paper, according tofactors relatively strong affectingthe PVoutput.

Set $X=\left\{x_{1}, x_{2}, \ldots, x_{k}\right\}$ as the historical sample, and set $x_{i}(i=1,2, \ldots k)$ as a single sample. $x_{i}(i=1,2, \ldots k)$ is a vector including all weather data of one day.kis the number of historical days. There are $\mathrm{m}$ factors, and xi can be expressed as a vector $x_{i}=\left(x_{i 1}, x_{i 2}, \ldots, x_{i m}\right)$. Because differentmeteorologicalfactors impactPVoutput in different level, so we give appropriateweighting factorforeach factor. In this paper,takingEuclidean distancemethod to calculate:

$$
d_{i}=\sqrt{\sum_{j=1}^{m} \lambda_{j}\left(x_{i j}-x_{j}\right)^{2}}
$$

Where: misthe number of influence factors; $\lambda j$ is thecorrelation coefficient calculated by equation (2); $X_{i j}$ is i-th day's $j$-th meteorological factor; $x j$ is the $j$-th meteorological factor of the day to be predicted.

When di is smaller,the association is stronger. Select threelargestcorrelationdays as the similar days. The meteorological and PV output data of the similar days constitute the training and testing samples, acting as SVM model input as well.

\section{SUPPORT VECTOR MACHINE AND PARAMETER OPTIMIZATION}

\section{A. Support Vector Machine}

SVMs are statistics learning tools introduced by Vapnik in 1995, these are usually used in classification and regression problems. SVR algorithm is mainly used v-SVR $\varepsilon$-SVR and LS-SVR[9-10],etc.\&-SVR requires less parameters and has good generalization performance [10], which make it the prediction model used in this article. $\varepsilon$-SVR is as follows:

$$
\begin{array}{ll}
\min & \frac{1}{2}\|w\|^{2}+C \frac{1}{l} \sum_{i=1}^{l}\left(\xi_{i}+\xi_{i}^{*}\right) \\
\text { s.t. } & y_{i}-w \phi\left(x_{i}\right)-b \leq \varepsilon+\xi_{i}, \xi_{i} \geq 0 \\
& w \phi\left(x_{i}\right)+b-y_{i} \leq \varepsilon+\xi_{i}^{*}, \xi_{i}^{*} \geq 0
\end{array}
$$

The model can be written as:

$$
f(\mathrm{x})=\sum_{i=1}^{N} y_{i}\left(\alpha_{i}-\alpha_{i}^{*}\right) k\left(x_{i}, x\right)+b
$$

Where $w$ is a vector of weights, and $b$ a constant. The slack variables $\xi_{i}, \xi_{I}^{*}$ are introduced to compensate the possible presence of excessive noise or outliers. So $\xi_{I}$ and $\xi_{I}^{*}$ are the positive and negative error respectively. The positive constant $C$ is a hyper-parameter adjusting the compromise between the amounts authorized error and the flatness of the function $f$.

The function $k$ is called kernel function; sigmoidal and radial basis function (RBF) defined as follows:

$$
K\left(x_{i}, x\right)=\exp \left(-\left\|x_{i}-x\right\|^{2} / 2 p^{2}\right)
$$

In summary, parameters $C$, $\varepsilon$ and $p$ are the key parameters that affect SVM prediction performance, and therefore it needs to select the optimum parameters.

\section{B. Parameters Optimization}

The currentmethods of determining the parametersare:experience/experiment select method[11-13], genetic algorithm [14],gridsearch and 
otheroptimizationalgorithms.Grid

searchcantraverseall possiblecombinations of parameters, having advantages to solve small-sample forecasting problems.Therefore, we used a two-stage method to determine $\varepsilon$-SVRparameters: firstly, using a gridsearchto determinesand possiblerange of $\mathrm{C}$ and $\mathrm{p}$; secondly, usingSADEforC and p optimization.

The data fromFebruary 1 to March 31were the trainingsamples, the data from February 1 to February 8 were the testsamples.Set ( $\varepsilon, \mathrm{p}, \mathrm{C}$ ) asoptimizationvariable to design grid searchtest.Setewithinthe range of0.001 to 0.01 , changingin steps of 0.001 .Set pin the range of0.1 to 2, changinginsteps of 0.1 . Set $\mathrm{C}$ withinthe range of0.1 to 10 , changing in stepsof 0.5. A three-dimensional grid was formed. RMSE(Root Mean Squared Error)was calculatedas follows:

$$
R M S E=\sqrt{\frac{1}{n} \sum_{i=1}^{n}\left(y_{i}-y_{i}^{\prime}\right)^{2}}
$$

Where, $\mathrm{n}$ is the number of all parameter groups, yi is the predicted values, $\mathrm{yi}^{\prime}$ is the true values.

Project RMSEcurveontoeplane. The results that RMSEchanging withe are shown in Figure1.

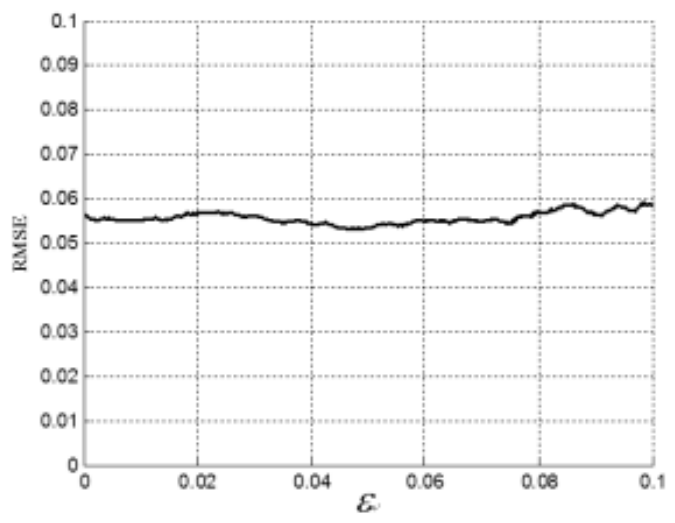

FIGURE I. RMSE VALUES CHANGE WITH $\varepsilon$.

Figure 1 shows that, when the parametercchanges, RMSE are inbetweenfrom 0.05 to 0.06. RMSEchanges stably, meaning the impact of on theperformance of the model is not significant. Knownfrom the literature [14], when p and Care fixedon asuitablevalue range, SVR performance is not sensitive to $\varepsilon$.

\&controls thesparsenessof support vectors. The larger $\varepsilon$ is,the fewersupport vectors are. When $\varepsilon$ exceeds a certain figure, lesslearning phenomenon will exist, increasing the prediction error.So we make $\varepsilon=0.01$ in test 2 .

Set ( $\mathrm{p}, \mathrm{C}$ ) asoptimizationvariable to design grid searchtest again. Setcto0.01.Set pinthe range of0.1 to 2, in steps of0.1. SetCin therangeof 0.1 to 10,insteps of 0.5.RMSEis calculatedin the same way, and results are shown in Figure2.

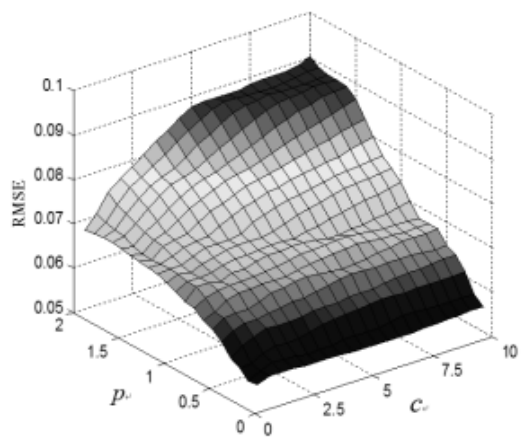

FIGURE II. RMSE VARY WITH P AND C.

Sowe determined the appropriaterangeof $\mathrm{C}$ andpthrough thegrid search, and used self-adapting differentialevolution (SADE) [15] algorithm to select optimum figure.

UsingSADE algorithm to generateinitial population randomly, the number of individualsis 20.Then calculatethe fitness ofthe initial populationof individuals. New offspring individuals were generated in solution space by mutationandcrossover strategybased ondifferential evolution algorithm[16], evaluated by fitness. Offspringandparentpopulations were selected by greedy algorithm, based on individual fitness. Aftera certainnumber of iterations, we got the bestC andp,making the structural risk minimum.

\section{IV.CASE STUDY AND RESULTS}

The output data of the roof-mounted PV power plant and the light, temperature datain the region from February 1, 2013toMarch 31were acted as test data. Two methods were applied in the case. One is the FCA-GS/SADE-SVRmodel proposed above, another is a typical traditionalstatisticalmethod-back propagation (BP)neural networkforecastingmethod. The comparativeresults are shown in Figure3 4.

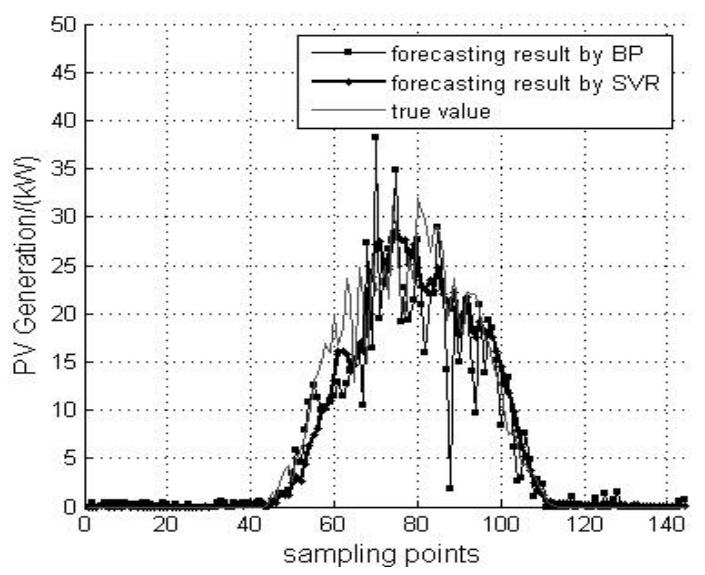

FIGURE III. COMPARISON OF PREDICTION RESULT USING BP AND FCA-GS/SADE-SVR. 


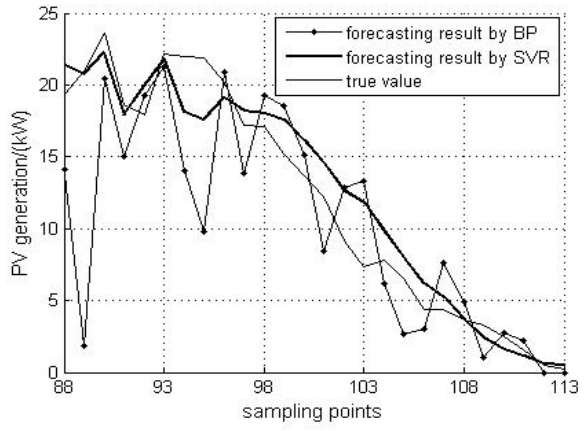

FIGURE IV. PARTIAL COMPARING FIGURE OF PREDICTION.

Multiplecomparison tests were proceeded by the two methods, getting the averageRMSE and illustrating as follows:

TABLE II. AVERAGE RMSE OF TEST RESULT.

\begin{tabular}{|c|c|c|c|c|c|c|c|c|}
\hline & $\begin{array}{c}\text { For } \\
\text { ecas } \\
\mathbf{t} \\
\mathbf{d a y} \\
\mathbf{1}\end{array}$ & $\begin{array}{c}\text { For } \\
\mathbf{e c a s} \\
\mathbf{t} \\
\mathbf{d a y} \\
\mathbf{2}\end{array}$ & $\begin{array}{c}\text { For } \\
\mathbf{e c a s} \\
\mathbf{t} \\
\mathbf{d a y} \\
\mathbf{3}\end{array}$ & $\begin{array}{c}\text { For } \\
\mathbf{e c a s} \\
\mathbf{t} \\
\mathbf{d a y} \\
\mathbf{4}\end{array}$ & $\begin{array}{c}\text { For } \\
\mathbf{e c a s} \\
\mathbf{t} \\
\mathbf{d a y} \\
\mathbf{5}\end{array}$ & $\begin{array}{c}\text { For } \\
\mathbf{e c a s} \\
\mathbf{t} \\
\mathbf{d a y} \\
\mathbf{6}\end{array}$ & $\begin{array}{c}\text { For } \\
\mathbf{e c a s} \\
\mathbf{t} \\
\mathbf{d a y} \\
\mathbf{7}\end{array}$ & $\begin{array}{c}\text { For } \\
\mathbf{e c a s} \\
\mathbf{t} \\
\mathbf{d a y} \\
\mathbf{8}\end{array}$ \\
\hline BP & 8.9 & 7.29 & 7.37 & 7.41 & 4.88 & 3.72 & 9.01 & 5.64 \\
\hline $\begin{array}{c}\text { FCA- } \\
\text { GS/SA } \\
\text { DE- } \\
\text { SVR }\end{array}$ & 7.28 & 4.86 & 6.58 & 2.84 & 4.55 & 2.98 & 9.7 & 4.62 \\
\hline
\end{tabular}

Known fromTable 2, Figure3 and Figure 4, the method proposed in this paper can describe PV output short-term characteristics more accurately except the seventh day.The averageRMSE of 8 days calculated by BPpredictionis6.789\%. While using SVRmethod, the figure is 5.551\%, and improving $1.238 \%$ by contrast.

\section{CONCLUSIONS}

This paper analyzes theactual output data of distributedPV andlocalweather data, selectinglight intensity and temperature as the relativestrong correlation factors.Based ontwo relatedfactors, fuzzy clusteringtheory is applied to selectsimilardays.A two-stage method is proposed to determinec-SVRparameters. Case showsthat the FCA-GS / SADE-SVRmethodcandescribe characteristicsof PVoutputinshort-term more accuratelythan the BPneural networkforecastingmethod.

\section{ACKNOWLEDGEMENTS}

This work was supported by the National Key Technology R\&D Program of China (No.2013BAA02B02).

\section{REFERENCES}

[1] Tan Y, Meegahapola L, Muttaqi K M. A review of technical challenges in planning and operation of remote area power supply systems[J]. Renewable and Sustainable Energy Reviews, 38: 876-889, 2014.

[2] Brouwer A S, van den Broek M, Seebregts A, et al. Impacts of largescale Intermittent Renewable Energy Sources on electricity systems, and how these can be modeled[J]. Renewable and Sustainable Energy Reviews, 33: 443-466, 2014.

[3] MaoMeiqin, GongWenjian, ZhangLiuchen, etc. Short-term photovoltaic generation forecasting based on EEMD-SVM combined
method[J].Proceedings of The CSEE, 33(34): 17-24, 2013.

[4] MaoMeiqin, GongWenjian, ZhangLiuchen, etc. Short-term photovoltaic generation forecasting based on EEMD-SVM combined method[J].Proceedings of The CSEE, 33(34): 17-24, 2013.

[5] Bouzerdoum M, Mellit A, MassiPavan A. A hybrid model (SARIMASVM) for short-term power forecasting of a small-scale grid-connected photovoltaic plant[J]. Solar Energy, 98: 226-235, 2013.

[6] Ren Hong, Ye Lin. Operation characteristics of PV system under the influence of environmental factors[J].Transactions of China Electrotechnical Society, (10): 158-165, 2010.

[7] Zhang Xueli,LiuQihui,MaHuimeng,LiBei[J]Power System and Clean Energy,28(5): 76-81, 2012.

[8] Zhang Jiawei, Zhang ZijiaShort-term photovoltaic system power forecasting based on PSO-BP neural network[J]Renewable Energy Resources, 8:006,2012.

[9] Vapnik V N.The Nature of Statistical Learning Theoty.Berlin:SpringerVerlag,1995.

[10] Vapnik V N,GolowichS,SmolaA.Support vector machine for function approximation, regression estimation and signal processing//Michael C,etal,ed. Advances in Neural Information Processing Systems 9,Cambridge,MA:Massachusetts Institute of Technology Press, 1997.

[11] LanHua,LiaoZhimin, Zhao Yang. ARMAmodelof the solar power station based on output prediction[J].Electrical Measurement \& Instrumentation,(2): 31-35, 2011.

[12] Safie F M . Probabilistic Modeling of Solar Power Systems[C] . Atlanta, GA : Reliability and Maintain ability Symposium, 1989.

[13] Muselli M, Poggi P, Notton G, et al. First order Markov chain model for generating synthetic "typical days" series of global irradiation in order to design photovoltaic stand-alone systems[J]. Energy Conversion and Management, 42(6): 675-687, 2001.

[14] Ding Ming, ZhouNing. A method to forecast short-term output power of photovoltaic generation system based on Markov Chain[J].Power System Technology,35(1): 152-157, 2011.

[15] Brest J, Greiner S, Bošković B, et al. Self-adapting control parameters in differential evolution: a comparative study on numerical benchmark problems[J] . IEEE Transactions on Evolutionary Computation, 10(6): 646-657, 2006.

[16] Advances in differential evolution[M]. Springer Verlag, 2008. 